\title{
Startup Dynamic Behaviour of a Jeffcott Rotor
}

\author{
H. Diken and K. Alnefaie \\ King Abdulaziz University, Mechanical Engineering Department, P.O. Box 80204, Jeddab, 21589, Saudi Arabia
}

(Received 3 October 2004; accepted 4 May 2005)

\begin{abstract}
In this study, the dynamic behaviour of a Jeffcott rotor during startup is analysed. Equations of a Jeffcott rotor model are obtained assuming that rotor speed is not constant. Startup speed is assumed to have a second order control system transfer function. Numerical calculations show that if the rotor is running in a subcritical condition, maximum whirl radius is reached before the rotor reaches the critical speed. If the rotor is running in a supercritical condition, the maximum whirl radius is reached after the rotor passes the critical speed. An overshoot of the startup speed significantly increases the value of the maximum whirl radius. It is also observed that, for a supercritical startup, the whirl radius has a cyclic behaviour before it reaches a steady state value.
\end{abstract}

\section{INTRODUCTION}

Most of the rotor dynamic analyses are performed under the hypothesis of a constant angular speed without taking into account the terms associated with angular acceleration. Pioneering studies of acceleration through critical speeds were made by Lewis ${ }^{1}$, Baker ${ }^{2}$, Mauer and Weibel ${ }^{3}$. They looked at the problem from an analytical point of view, employing a force which is independent of the speed. Lee et al. ${ }^{4}$ presented a finite element formulation that permits the analysis of the transitory response during the acceleration or deceleration of an asymmetric rotor-bearing system. Ishida and Inoue $^{5}$ investigated nonstationary oscillation of the Jeffcott rotor with nonlinear spring characteristics during acceleration through the major critical speed, theoretically and experimentally. Hassenpflug et al. ${ }^{6,7}$ and Gasch et al. ${ }^{8}$ have studied the influence of acceleration on the critical speed of a Jeffcott rotor. In these studies, constant acceleration is assumed during startup. It is shown that the absolute maximum occurs at an angular velocity greater than the critical speed of the rotor. For supercritical cases, a beating phenomenon is also observed.

Shi et al. ${ }^{9}$ presented an adaptive time-frequency decomposition technique to describe the transient vibration of rotating machinery. Their results prove that the approach can specify critical speed as well as acceleration rates accurately and is also effective in ensuring that machinery can pass through the critical speed safely. Gu et al. ${ }^{10}$ extended the transfer matrix technique to a transient response analysis of a large complex nonlinear rotor bearing system by applying a transfer matrix-Newark formulation. This new formulation proves to be effective on transient response calculations.

In this study, equations of a Jeffcott rotor model are developed assuming that the speed is not constant. Electrical motors generally have a second order speed control system. It is assumed that the rotor startup speed increases to a maximum steady speed according to a second order speed control transfer function. Nondimensional equations are solved numerically. The effect of startup speed overshoot and speed control system damping on the whirl radius is analysed.

\section{FORMULATION}

Figure 1 shows the cross sectional view of the Jeffcott rotor. The Jeffcott rotor is comprised of a relatively thin disk carried at the middle by an elastic shaft, which is simply supported. Here, $0 x y$ is the fixed coordinate system, 0 is the undeflected centre of the rotor, $S$ is the rotor centre after deflection, $G$ is the mass centre, $r$ is the amount of deflection of the flexible shaft, $e$ is the mass eccentricity, $\theta$ is the whirl angle, and $\varphi$ is the disk rotation. Equations of motion, after applying Newton's law, give ${ }^{11}$

$$
\begin{aligned}
& m \frac{d^{2}}{d t^{2}}(x+e \cos \varphi)=-k x-c \dot{x} ; \\
& m \frac{d^{2}}{d t^{2}}(y+e \sin \varphi)=-k y-c \dot{y},
\end{aligned}
$$

where $m$ is the disk mass, $x$ and $y$ are the deflections in the $x$ and $y$ directions respectively, $k$ is the shaft stiffness, and $c$ is the elastic shaft viscous damping. After derivations, Eq. (1) will have the following form,

$$
\begin{aligned}
& m \ddot{x}+c \dot{x}+k x=m e \dot{\varphi}^{2} \cos \varphi+m e \ddot{\varphi} \sin \varphi ; \\
& m \ddot{y}+c \dot{y}+k y=m e \dot{\varphi}^{2} \sin \varphi-m e \ddot{\varphi} \cos \varphi .
\end{aligned}
$$

After using some definitions, the equations are

$$
\begin{aligned}
& \ddot{\bar{x}}+2 \zeta \omega_{n} \dot{\bar{x}}+\omega_{n}^{2} \bar{x}=\dot{\varphi}^{2} \cos \varphi+\ddot{\varphi} \sin \varphi ; \\
& \ddot{\bar{y}}+2 \zeta \omega_{n} \dot{\bar{y}}+\omega_{n}^{2} \bar{y}=\dot{\varphi}^{2} \sin \varphi-\ddot{\varphi} \cos \varphi,
\end{aligned}
$$

where

$$
\bar{x}=\frac{x}{e}, \bar{y}=\frac{y}{e}, \frac{c}{m}=2 \zeta \omega_{n}, \frac{k}{m}=\omega_{n}^{2} .
$$

Assuming nondimensional time $\tau=\omega_{n} t$, the following equations are obtained,

$$
\begin{aligned}
& \bar{x}^{\prime \prime}+2 \zeta \bar{x}^{\prime}+\bar{x}=\frac{\varphi^{\prime 2}}{\omega_{n}^{2}} \cos \varphi+\frac{\varphi^{\prime \prime}}{\omega_{n}^{2}} \sin \varphi ; \\
& \bar{y}^{\prime \prime}+2 \zeta \bar{y}^{\prime}+\bar{y}=\frac{\varphi^{\prime 2}}{\omega_{n}^{2}} \sin \varphi-\frac{\varphi^{\prime \prime}}{\omega_{n}^{2}} \cos \varphi,
\end{aligned}
$$

where $\bar{x}^{\prime}=\omega_{n} \dot{\bar{x}}, \bar{x}^{\prime \prime}=\omega_{n}^{2} \ddot{\bar{x}}$ and $\bar{y}^{\prime}=\omega_{n} \dot{\bar{y}}, \bar{y}^{\prime \prime}=\omega_{n}^{2} \ddot{\bar{y}}, \zeta$ is the damping ratio, and $\omega_{n}$ is the natural frequency of the Jeffcott rotor. Since the speed is not constant, Eq. (2) has an additional term having acceleration $\ddot{\varphi}$. 